\title{
CORE OVERSHOOTING UNDER THE LIGHT OF FLUID DYNAMICS
}

\author{
M. Rieutord ${ }^{1}$
}

\begin{abstract}
We discuss the possible contraints that are brought about by a fluid mechanical analysis of the overshooting phenomenon at the interface of convective cores and radiative envelopes of early-type stars. We investigate an improvement of Roxburgh's criterion by taking into account the viscous dissipation but show that this criterion remains not stringent enough to be predictive. We then discuss the thickness of the overshooting layer and show that all estimates, including the one of Zahn (1991), lead to a very thin mixing layer typically less than a percent of the pressure scale height.
\end{abstract}

\section{Introduction}

Overshooting and penetrative convection are describing the extension of stellar convective regions beyond the limits predicted by the Schwarzschild criterion. We recall that this criterion says that the fluid is convectively unstable as soon as the actual temperature gradient exceeds (in absolute value) the adiabatic gradient. Along a similar line, the mixing-length approach of stellar convection assumes that potential energy is locally transformed into kinetic energy. According to this simple model, convection ceases as soon as the driving ceases. Hence, convective flows should not go beyond the boundaries given by the Schwarzschild criterion. This property of the mixing-length theory is of course a shortcoming of the model since inertia of the fluid let the flows go beyond the driving region. Previous work work (e.g. Browning et al. 2004, and references therein) have defined penetrative convection as the extent of convective region where the temperature gradient remains adiabatic, while the overshoot layer is the place mixed by flows triggered by a neighbouring convective flow. This layer is beyond the region of penetrative convection and endowed with a stable temperature gradient.

${ }^{1}$ Université de Toulouse; UPS-OMP; IRAP; Toulouse; France and CNRS; IRAP; 14, avenue Edouard Belin, 31400 Toulouse, France 
Penetrative convection and overshooting have a different impact on stars. In addition to extending the mixed fluid beyond the Schwarzschild boundary, penetrative convection slightly modifies the stellar structure by extending the quasiconstant entropy regions of the star. Overshooting is thought as a mixing process not efficient enough to impose an adiabatic temperature gradient, but which impacts a larger region, beyond the convective penetration. To simplify the wording, we shall call hereafter the extension of the flows beyond the Schwarzschild limit simply as overshooting, and make the distinction with penetrative convection when necessary.

From the point of view of stellar evolution, overshooting of convective cores is crucial. Through the induced mixing, it makes a larger amount of material available to nuclear reactions, which are active near the centre of the star. The immediate consequence is that the main sequence of stars owning a convective core (the so-called early-type stars) last longer than the duration predicted by models not including overshoot. Although this effect was first thought to be negligible (Saslaw \& Schwarzschild 1965), detailed observations have accumulated evidences in favour of an effective presence of core overshooting. Mixing material beyond the Schwarzschild boundary of a convective core is necessary to explain the isochrone of stellar clusters, the common birth of eclipsing binaries or the spectrum of eigenmodes in SPB stars (Moravveji et al. 2015).

While the Schwarzschild boundary is easy to predict, the right way of finding the extension of the overshooting region is still an open question. Basically two recipes are currently used in 1D stellar models: (i) the core is plainly extended by some fraction of the local pressure scale height (typically 20\%), or (ii) the neighbouring radiative layer is endowed with a turbulent diffusion that decays exponentially with the distance to the core boundary. This latter recipe was originally proposed by Freytag et al. (1996) after the first hydrodynamical investigation of overshooting. These recipes help interpret the data but have no predictive power. We still cannot predict how overshooting varies, with the mass, the age or the metallicity of stars.

A possible way of investigating the properties of overshooting layers is the use of direct numerical simulations. Such 3D simulations are not very common. After the pioneering work of Freytag et al. (1996) in two-dimensions, 3D simulations of convective overshoot in a belowing layer was undertaken by Brummell et al. (2002) who did not observe any penetration but just overshoot. Then, Browning et al. (2004) investigated core overshooting of an A-star with 3D simulations in spherical geometry. They noted the difficulty of precisely defining the size of the overshooting layer. More recently, Gilet et al. (2013) also considered overshooting through $3 \mathrm{D}$ simulations of core convection. In this work the authors show that mixing between the stable radiative envelope and the convective core occurs through turbulent entrainment thus underlining the importance of shear instabilities at this interface.

In the following we shall first review the approach of Roxburgh (1989) and propose an improvement to this analysis that include viscous dissipation (Sect. 2). We then present three phenomenological analysis of the dynamics in the layer and 
show that all lead to a very thin mixing layer (Sect. 3). Some perspectives end the paper.

\section{Reviewing the theory}

\subsection{No theory: Prescriptions}

In most evolution codes, core overshooting is taken into account via one of the two following prescriptions. The first one is the so-called "step overshooting" (Moravveji et al. 2015) where the size of the core is just extended, via a step function, by some fraction of the pressure scale height, namely $\delta r=\beta H_{p}$. This extension of the core enlarges the adiabatic part of the star (since convective cores are almost isentropic).

The second prescription is the so-called "exponential overshooting" where mixing beyond the Schwarzschild boundary of the core is assumed to be due to some turbulent diffusion, modelled by a turbulent diffusivity of the form:

$$
D_{\text {ov }}(z)=D_{c} \exp \left(-\frac{2 z}{f_{\mathrm{ov}} H_{p}}\right)
$$

where $D_{c}$ is a diffusion coefficient and $z=r-R_{\text {core }}$. For stars with $3.13 M_{\odot} \leq$ $M \leq 3.25 M_{\odot}$ and $Z \in[0.014,0.028]$, Moravveji et al. (2015) found that seismic data are well fitted with $f_{\text {ov }} \sim 0.0175$ and $1.75 \leq \log D_{c} \leq 2$ with $D_{c}$ in $\mathrm{cm}^{2} / \mathrm{s}$. These authors find that the exponential overshooting gives better results than step overshooting.

\subsection{Roxburgh criterion}

Let us now briefly present the first criterion that has been proposed given some general properties of fluid flows in the overshooting layer, namely Roxburgh criterion (Roxburgh 1989). The idea is to start from the entropy equation of fluid mechanics, namely

$$
\rho T \frac{D s}{D t}=\operatorname{div}(\chi \vec{\nabla} T)+\mathcal{D}+\rho \varepsilon
$$

$\chi$ is the heat conductivity and $\varepsilon$ the energy production per unit mass. After some manipulation of this equation and integration over a volume (V) limited by a surface $(\mathrm{S})$ that includes the core, we get

$$
\int_{(S)}\langle\rho s \vec{v}\rangle \cdot d \vec{S}=\int_{(V)}\left\langle\frac{\operatorname{div}(\chi \vec{\nabla} T)+\mathcal{D}+\rho \varepsilon}{T}\right\rangle d V
$$

where we considered a statistically steady state of the fluid flows and where $\langle\cdot\rangle$ indicates an ensemble average.

We now consider a non-rotating non-magnetic star, spherically symmetric in its steady state. We introduce $\vec{F}_{r}$ and $\vec{F}_{n}$, respectively the radiative flux insured 
by the diffusion of photons, and the flux produced by the nuclear reactions. They are such that $\vec{F}_{\mathrm{r}}=-\chi \vec{\nabla} T$ and $\operatorname{div} \vec{F}_{\mathrm{n}}=\rho \varepsilon$. Because of spherical symmetry we can express the total and radiative luminosities as $L(r)=4 \pi r^{2} \vec{F}_{\mathrm{n}} \cdot \vec{e}_{r}$ and $L_{r}(r)=$ $4 \pi r^{2} \vec{F}_{\mathrm{r}} \cdot \vec{e}_{r}$. In the core the difference, $L_{c}=L-L_{r}$, is the convective luminosity. Taking the bounding surface $(S)$ as a sphere of radius $r_{0}$ where temperature is $T_{0}$ and located well outside the energy production region, (2.3) may be written:

$$
\frac{T_{0}}{L_{*}} \int_{0}^{r_{0}}\left\langle L_{c}\left(T^{-1}\right)^{\prime}\right\rangle d r=\frac{L_{*}-L_{r}-4 \pi r_{0}^{2} T_{0}\left\langle\rho s v_{r}\right\rangle}{L_{*}}+\frac{T_{0}}{L_{*}} \int_{(V)}\left\langle\frac{\mathcal{D}}{T}\right\rangle d V
$$

where $L_{*}$ is the luminosity of the star.

Beyond the core and beyond the overshooting flows the radiative luminosity just equals the total luminosity of the star, while the radial entropy flux is presumably negligible. If we further neglect the second order correlations, then we may further simplify (2.4) into the following final form:

$$
\int_{0}^{r_{0}}\left\langle L_{c}\right\rangle\left(\langle T\rangle^{-1}\right)^{\prime} d r=\int_{(V)} \frac{\langle\mathcal{D}\rangle}{\langle T\rangle} 4 \pi r^{2} d r
$$

\subsection{Discussion of Roxburgh's criterion}

The transformation of (2.5) into a criterion on core overshooting is obtained after two further assumptions. First we need to specifiy the value of the temperature gradient in the overshooting region. We know that this gradient is subadiabatic but if the mixing is vigorous enough it can be assumed adiabatic. Let us adopt this limiting case. The second assumption is to neglect, altogether the RHS of (2.5). With this last assumption, every term is known and $r_{0}$ need to be searched as the first zero of the non-dimensional function

$$
F(r)=\frac{T_{0}}{L_{*}} \int_{0}^{r}\left\langle L_{c}\right\rangle\left(\langle T\rangle^{-1}\right)^{\prime} d x .
$$

As an illustration we show in Figure 1 a plot of the function $F(r)$ for a $3.25 M_{\odot}$ CESAM model of the star KIC10526294. In this case the predicted extension of the core with Roxburgh criterion is $71 \%$ of the local pressure scale height. Other values computed for different masses are shown in Table 1 . There we see that the overshooting distance computed with this model is much larger than the expected values derived from comparison to observations $\left(0.2 H_{p}\right)$. This is a well-known result (Maeder 2009).

\subsection{Estimates of the viscous dissipation}

Roxburgh (1989) insisted on the fact that the value of $r_{0}$ defined as the first zero of $F(r)$, is just an upper bound of the actual outer radius of the overshoot layer. Obviously this upper limit is not stringent. In view of the list of approximations that have been made to derive (2.6), we may wonder if taking into account the 


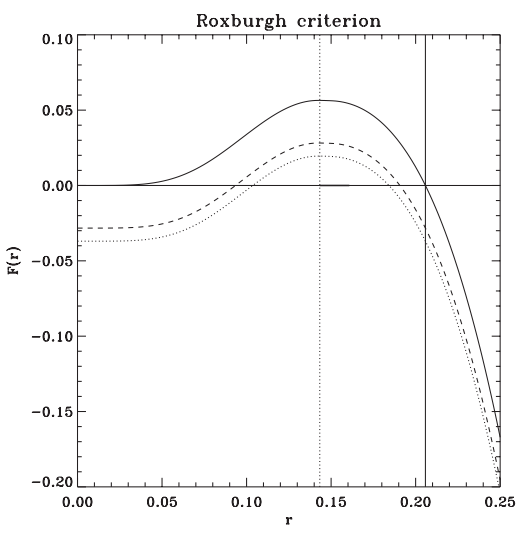

Fig. 1. Solid line: $F(r)$-function (2.6) for the $3.25 M_{\odot}$ model (see Table 1). The vertical dotted line marks the position of the Schwarzschild limit, and the solid vertical line marks the limit of the overshooting region according to Roxburgh criterion. Effect of dissipation $D_{1}$ (dotted) and $D_{2}$ (dashed) models are also shown. The thick segment shows a $0.2 H_{p}$ overshoot.

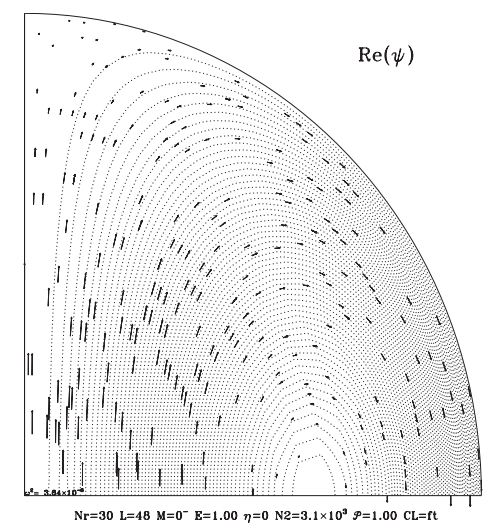

Fig. 2. Meridional flow of the convection cell that appears at the threshold of instability in a sphere when the fluid is heated by a uniform distribution of heat sources.

viscous dissipation gives a more stringent criterion. The new function to consider is

$$
F(r)=\frac{T_{0}}{L_{*}} \int_{0}^{r}\left\langle L_{c}\right\rangle\left(\langle T\rangle^{-1}\right)^{\prime} d x-\frac{T_{0}}{L_{*}} \int_{0}^{r} \frac{\langle\mathcal{D}\rangle}{\langle T\rangle} 4 \pi r^{2} d r .
$$

We note that since the viscous dissipation is strictly positive, its effect is indeed to reduce the value of $r_{0}$ and to give a more stringent criterion.

There are various ways of estimating the power dissipated by viscosity in a turbulent flow. Let us now discuss some possibilities. 
Table 1. Overshooting distance according to Roxburgh's criterion without viscous dissipation for a few stellar models.

\begin{tabular}{ccccc}
\hline Mass & Code & Age (Myrs) & $\mathrm{r}_{\text {Schw }}$ & $d_{\text {Rox }}^{\text {invisc }} / H_{p}$ \\
\hline 1.5 & MESA & 0 & 0.093 & 0.26 \\
3 & MESA & 0 & 0.138 & 0.53 \\
3 & CESAM & 0 & 0.151 & 0.58 \\
5 & MESA & 0 & 0.171 & 0.67 \\
5 & CESAM & 0 & 0.174 & 0.62 \\
3.25 & CESAM & 60 & 0.143 & 0.71 \\
\hline
\end{tabular}

\subsubsection{A mixing-length argument}

In homogeneous, statistically steady turbulence of an incompressible fluid, we know (Davidson 2004) that turbulent dissipation, $\langle\varepsilon\rangle$ in Kolmogorov theory, is related to the most energetic scale, the integral scale $\ell_{0}$, by

$$
\langle\mathcal{D}\rangle=\rho\langle\varepsilon\rangle=\rho C_{\varepsilon} \frac{v^{3}}{\ell_{0}}
$$

where $v$ is the rms velocity of the most energetic eddies. $C_{\varepsilon}$ is an a priori universal constant of homogeneous isotropic turbulence whose value is around 0.5 (Vassilicos 2015).

In the mixing-length approach of stellar convection we may identify $v$ with the largest value of the mixing-length velocities and $\ell_{0}$ with the mixing length itself. We recall that in the mixing length scenario, a fluid parcel reaches a velocity $v$ after the work of the buoyancy over a distance equal to the mixing length.

The difficulty is here to evaluate the ratio $v^{3} / \ell_{0}$. If $\ell_{0}$ is the mixing-length, in the regime of efficient convection ${ }^{1}$ (high Péclet number), this ratio is independent of the mixing-length; actually

$$
\frac{v^{3}}{\ell_{0}}=\left(\nabla_{r}-\nabla_{a d}\right) \frac{g^{2} \rho \kappa \delta}{4 P}
$$

However, this ratio is still plagued by the limitations of the MLT. It is local and zero where the driving is zero. For instance at the centre of the core, it predicts zero viscous dissipation (gravity vanishes there), which is certainly not the case.

We shall therefore set the velocity $v$ to the maximum velocity given by the mixing-length model, which we expect to be the typical velocity of the most energetic structures (those where the buoyancy has worked the most!). As for the integral scale $\ell_{0}$, we'll set it to the radius of the core which is the largest possible length for an eddy.

\footnotetext{
${ }^{1}$ In convective cores, the mixing-length parameter $U$ is very small, typically $\sim 10^{-10}$, signing for a very effective convection regime.
} 
We recall that small amplitude convection in a full sphere, driven by internal heating, leads to an $\ell=1$ (in the spherical harmonic expansion) cell that has maximum velocity at the centre of the sphere (see Fig. 2). Even if core convection is expected to be highly turbulent, it is plausible that the most energetic structures have a size comparable to that of the core and that much energy is borne by the $\ell=1$ "mode", as actually suggested by the simulations of Gilet et al. (2013) and their Figure 5a.

Thus we tested two expressions for the dissipation. The first is simply to say that

$$
\langle\mathcal{D}\rangle=D_{1}=\rho C_{\varepsilon} \frac{v_{\max }^{3}}{r_{\text {core }}}
$$

while the second takes the maximum value of the MLT prediction (2.9) for $v^{3} / \ell_{0}$, so

$$
\langle\mathcal{D}\rangle=D_{2}=\rho C_{\varepsilon} \max _{\text {over the core }}\left(\left(\nabla_{r}-\nabla_{a d}\right) \frac{g^{2} \rho \kappa \delta}{4 P}\right)
$$

We tested the Roxburgh criterion including viscous dissipation with the two foregoing dissipation models, using the recently analysed, slowly rotating SPB star KIC10526294 of Moravveji et al. (2015).

Figure 1 shows where the function $F(r)$ of (2.7) first vanishes beyond the core radius as given by the Schwarzschild criterion. Without any dissipation, Roxburgh criterion gives an overshoot layer that extends over $0.715 H_{p}$. When dissipation model $D_{1}$ is included this thickness is reduced to $0.47 H_{p}$. When the second model $D_{2}$ is used, the layer is slightly thicker at $0.54 H_{p}$. The foregoing calculation shows that accounting of viscous dissipation makes Roxburgh's criterion slightly more stringent, although viscous dissipation is not precisely known. The ensuing question is: can we do better?

\subsubsection{Use of laboratory experiments}

Another possibility for evaluating viscous dissipation is to use the results of laboratory experiments. These experiments are usually in the Boussinesq regime where variations of density between top and bottom are of small amount. Nevertheless, if the core is not too big, this might be an acceptable solution. For instance, in a $1.5 M_{\odot}$ star, MESA gives a ratio $\rho_{s} / \rho_{c} \sim 1.3$ between the surface and central density of the core. This is not very large and Boussinesq approximation may be used as a first step.

Using this approximation, it may be shown that local dissipation in the bulk of the flow is given by

$$
\mathcal{D}=\rho \frac{\nu^{3}}{H^{4}}(\mathrm{Nu}-1) \mathrm{Ra} / \mathcal{P}^{2}
$$


$\mathrm{Nu}$ is the Nusselt number, Ra Rayleigh number and $\mathcal{P}$ the Prandtl number (Chillà \& Schumacher 2012). $H$ refers to the height separating the cooled and heated plates and $\nu$ is the kinematic viscosity.

From the foregoing expression we see that an evaluation of the Rayleigh number and of the height of the box are needed.

As far as the Rayleigh number is concerned, we shall follow Jones et al. (2009), who give the following definition for the Rayleigh number

$$
\mathrm{Ra}=\frac{G M d \Delta s}{\nu \kappa c_{p}}
$$

where $\Delta s$ is the entropy drop across the spherical shell of thickness $d$, which remains to be specified. The entropy gradient is

$$
\frac{\partial s}{\partial r}=\frac{c_{p} N^{2}}{g \delta} \quad \text { with } \quad \delta=-\left(\frac{\partial \ln \rho}{\partial \ln T}\right)_{P} .
$$

We assume $\Delta s=d \frac{c_{p} N^{2}}{g \delta}$ and hence

$$
\mathrm{Ra}=\frac{r^{2} d^{2} N^{2}}{\nu \kappa \delta}
$$

and we deduce

$$
\mathcal{D}=\rho \kappa N^{2} \frac{r^{2} d^{2}}{H^{4}} \mathrm{Nu}
$$

using $\mathrm{Nu} \gg 1 . H$ is the height of the experimental box. $d$ is the distance over which the unstable entropy gradient works. We thus choose $H=d=r$ so that

$$
\mathcal{D}=\rho \kappa N^{2} \mathrm{Nu}
$$

At this stage we may evaluate the Rayleigh number from (2.13) with $d=r$. It turns out that the maximum value reached over the convective core is $\sim 10^{20}$. We note that experiments of Chillà \& Schumacher (2012) have reached Rayleigh numbers of order $\mathrm{Ra} \sim 10^{17}$, which is not so far for the astrophysical value. With such experiments, Chillà \& Schumacher (2012) have obtained scaling laws for the heat flux as represented by the Nusselt number, namely $\mathrm{Nu} \simeq 0.05 \mathrm{Ra}^{1 / 3}$. Other experiments like the one of Niemela et al. (2000) find a similar scaling law namely $\mathrm{Nu} \simeq 0.124 \mathrm{Ra}^{0.309}$. Unfortunately, in these experiments, convection is forced by the boundary layers. Hence, the Nusselt tend to scale like $\mathrm{Ra}^{1 / 3}$ (e.g. Rieutord Rieutord 2015).

If the forcing is in the bulk, as it is by the nuclear heat sources in the core, Kraichnan (1962) and Spiegel (1971) have shown that we should expect $\mathrm{Nu}=$ $\mathrm{Nu}_{0}(\operatorname{Ra} \mathcal{P})^{1 / 2}$ also called the ultimate regime of convection, since in this case even the boundary layers are turbulent. In such a case, the Nusselt number is independent of $\nu$ and $\kappa$ and we actually retrieve the assumption used before, namely $\mathcal{D} \sim v^{3} / \ell_{0}$. Unfortunately, no laboratory experiment has reached this regime. 
Lohse \& Toschi (2003) have studied the ultimate regime numerically, but with $\mathrm{Ra} \in\left[10^{6}, 10^{7}\right]$. They find $\mathrm{Nu}_{0} \simeq 4 \times 10^{-3}$. Since in the core $\mathrm{Ra} \sim 10^{20}$ and $\mathcal{P} \sim 2 \times 10^{-6}, \mathrm{Nu} \sim 5.7 \times 10^{4}$, which we can combine with $(2.15)$ where we take the maximum value of $N^{2}$ over the core in order to avoid the vanishing of the dissipation at the centre. Summarizing we can take

$$
\mathcal{D} \sim 5.7 \times 10^{4} \rho \kappa N_{\max }^{3}
$$

Such an estimate leads to a dissipation power that is about ten times less than the previous estimates. However, it is likely that the law of Lohse \& Toschi (2003) is quite approximate with a too little range in Rayleigh number. Very recent experimental work on the ultimate regime by He et al. (2016) is also inconclusive because the explored range in $\mathrm{Ra}$ and $\mathcal{P}$ is too small.

\subsubsection{Discussion}

Roxburgh criterion combined with an estimate of the viscous dissipation inside the convective core was expected to give a more realistic constraint on the extension of the overshoot region in the radiative envelope of the star. The expected extent has been reduced but not to a value such that it can be used to make predictions with the models. The main result of the foregoing investigation is that viscous dissipation is not enough. Other terms need to be considered. These are the turbulent correlations and most probably their spatial variations within the overshoot layer.

\section{Approaching the local dynamics of the overshooting layer}

\subsection{Zahn's approach}

Let us now consider the approach of Zahn (1991) to the overshooting core. We still assume a spherical symmetry of the problem. In the core we have

$$
\frac{L}{4 \pi r^{2}}=F_{c}-\chi \frac{d T_{a}}{d r}
$$

if we assume the temperature gradient to be quasi-adiabatic. If we now assume that this is also true in the overshooting layer then we may write at first order that

$$
F_{c}(r)=F^{\prime}\left(r_{c}\right)\left(r-r_{c}\right)
$$

where $r_{c}$ is the core radius according to Schwarzschild's criterion. This equation just says that at the core radius the luminosity is wholly carried by radiative diffusion over the adiabatic gradient. It also says that beyond the Schwarzschild core radius, the convective flux has to be negative because the conductivity is high 
and the adiabatic gradient carries too much heat. Then, following Zahn (1991), we write $F_{c}=f \rho c_{p} V_{r} T^{\prime}$ and

$$
\frac{c}{2} \frac{d V_{r}^{2}}{d r}=\frac{T^{\prime}}{T} g \delta
$$

where $f$ and $c$ are $\mathcal{O}(1)$ dimensionless coefficients and $T^{\prime}$ is the amplitude of the temperature fluctuations. (3.3) is integrated replacing $T^{\prime}$ by its expression with respect to the convective flux, itself given by (3.2). Integrating from the core radius $r_{c}$ (given by Schwarzschild) to the limit of the overshooting region we obtain:

$$
r_{o}-r_{c}=(c f)^{1 / 2} V_{o}^{3 / 2} \sqrt{\frac{2 \rho c_{p} T}{3 g\left|F^{\prime}\left(r_{c}\right)\right| \delta}}
$$

where $V_{o}$ is the initial vertical velocity at $r_{c}$, in other words this is the velocity with which the fluid elements are launched into the stably stratified region. $c_{p}, T$ and $\rho$ are also taken at this place. The absolute value is needed because $F^{\prime}\left(r_{c}\right)<0$ since the convective flux is decreasing outwards. In (3.4), all quantities are well defined except $V_{o}$ and the product $c f$.

To evaluate $V_{o}$ we may proceed as follows. If the general mean flow is given by the cell shown in Figure 2, the vertical velocity at the boundary is given by turbulent entrainment, so that we can write $V_{o} \sim \alpha V_{h}$. The entrainment constant $\alpha$ is of order 0.1 (Rieutord \& Zahn 1995). We then remark that in such a cell the ratio between the central velocity and the surface velocity is $\sim 0.45$. The central velocity is however the maximum velocity in the convective cell. We shall identify this velocity with the maximum velocity that is given by the MLT in the core, on the argument that MLT gives the right order of magnitude for the flows. We therefore take $V_{o} \sim 0.045 V_{\text {MLT }}^{\max }$. With this evaluation and setting $c f=1$, we find that $\left|r_{o}-r_{c}\right| \sim 0.005 H_{p}$, leading to a very thing mixing layer.

\subsection{A layer at Richardson marginal stability}

We first explore another scenario that assumes the following hypotheses: (i) The core mean flow has the shape given by Figure 2, (ii) the interface is made unstable by the local shear, (iii) instabilities create a mixing layer of thickness $\delta$ and the layer is at marginal stability according to Richardson criterion.

Since entropy is minimum at the interface and since the Brunt-Väisälä frequency squared is proportional to the entropy gradient, at first order we have $N^{2}=\left(N^{2}\right)^{\prime} \delta$, where $\left(N^{2}\right)^{\prime}$ is the derivative of $N^{2}$ taken at the Schwarzschild radius. The shear $U^{\prime}$ is obviously of order $V_{s} / \delta$, where $V_{s}$ is the surface velocity of the cell. Marginal stability of Richardson criterion imposes that $U^{\prime 2} \sim 4 N^{2}$. Combining the foregoing relations gives the expression of the thickness of the shear layer, namely

$$
\delta^{3}=\frac{V_{s}^{2}}{4\left(N^{2}\right)^{\prime}}
$$


Taking for $V_{s}=0.45 V_{\text {mlt }}$ we find that $\delta \sim 0.002 H_{p}$ for our stellar model of $3.25 M_{\odot}$. This is again rather thin.

\subsection{A possible extension of the mixing layer}

However, within the shear layer small vortices are not sensitive to stratification if their scale is less than

$$
\ell=\sqrt{\frac{\kappa}{N}} \sim 3 \mathrm{~km}
$$

At this scale the velocity of the vortices should be related to that of the big vortices by

$$
v_{\ell}=(\ell / L)^{1 / 3} V_{L}
$$

according to the Kolmogorov cascade, $L$ being the scale of the large vortices (the integral scale). Small vortices can change the entropy profile as long as their Pclet number is larger than unity, namely

$$
v_{\ell} \ell / 3>\kappa .
$$

We look for the scale of the large vortices that is such that associated small vortices make a turbulent diffusion that overtakes heat diffusion. (3.7) and (3.8) yield

$$
\ell V_{L}(\ell / L)^{1 / 3}>3 \kappa
$$

which immediately shows that the scale of the large vortices is bounded if their velocity is bounded. Indeed, if they are too large the velocity they generate at the fixed scale (3.6), is too small and the ensuing turbulent diffusion cannot beat the heat diffusion of the fluid. Still noting that the Brunt-Väisälä frequency squared grows linearly with the scale, namely $N^{2}=\left(N^{2}\right)^{\prime} L$, we find that

$$
L=\left(V_{L} / 3\right)^{3 / 2}\left[\left(N^{2}\right)^{\prime} \kappa\right]^{-1 / 2}
$$

Taking $V_{L}=0.045 V_{\mathrm{MLT}}^{\max }$, we find $L \sim 0.001 H_{p}$ for our stellar model. So the order of magnitude of the thickness of the mixing layer is not changed by this additional phenomenon.

\section{Conclusions}

In this first study of the overshooting penetrative convection phenomenon we first assess the approach of Roxburgh (1989). We confirm what was already known, namely that Roxburgh criterion is a loose upper limit for the thickness of the overshooting layer. We tried to make this criterion more stringent by including the effects of viscous dissipation. We find that this implies a reduction of the thickness of the layer of $35 \%$ for the stellar model that we considered. So the criterion is more stringent but the evaluation of the viscous dissipation is difficult and 
therefore is an additional source of uncertainties. This investigation nevertheless underlines the importance of the global shape of the mean flow, which is not given by the mixing-length approach. We then focused on the dynamics of the overshooting layer, assuming that the convective flow in the core is in the first place looking like an $\ell=1$-cell. Evidence for the probable existence of such a mean flow comes from both laboratory (e.g. Tsuji et al. 2005) and numerical (Gilet et al. 2013) experiments. Assuming such a shape implies that the overshooting layer is mixed by shear instabilities rather than by the direct impingement of buoyant plumes. This leads to a very thin layer, at maximum a percent of the pressure scale height, that is totally mixed. Thus, the layer of penetrative convection is very thin according to this model. This means that the hydrostatic structure of the star is certainly not changed by this layer. This also means that the detection of such a layer by asteroseismology will be difficult.

The importance of core overshooting is however in the implied mixing between core and envelope. The next step is therefore an ab initio evaluation of the mixing rate at such an interface. We see three mechanism that are potentially contributing to this mixing: a large-scale flow in the radiative zone driven by the large-scale flow inside the core, turbulent diffusion by small eddies at the interface and last but not least, gravity or gravito-inertial waves excited by the interface. All these mechanisms require a detailed study to evaluate their relative importance and in fine their dependence with the global parameters of the star.

I am very grateful to Stéphane, Sacha, Corinne and Bérengère for the organization of this meeting, which reminded me the many enlightening and joyful discussions I had with Jean-Paul who had the secret of making science with pleasure.

\section{References}

Browning, M.K., Brun, A.S., \& Toomre, J., 2004, ApJ, 601, 512

Brummell, N.H., Clune, T.L., \& Toomre, J., 2002, ApJ, 570, 825

Chillà, F., \& Schumacher, J., 2012, Eur. Phys. J. E, 35, 58

Davidson, P. 2004, Turbulence: An introduction for Scientists and Engineers (Oxford University Press)

Freytag, B., Ludwig, H.-G., \& Steffen, M., 1996, A\&A, 313, 497

Gilet, C., Almgren, A.S., Bell, J.B., et al., 2013, ApJ, 773, 137

He, X., Bodenschatz, E., \& Ahlers, G., 2016, J. Fluid Mech., 791, R3

Jones, C.A., Kuzanyan, K.M., \& Mitchell, R.H., 2009, J. Fluid Mech., 634, 291

Kraichnan, R.H., 1962, Physics of Fluids, 5, 1374

Lohse, D. \& Toschi, F., 2003, Phys. Rev. Lett., 90, 034502

Maeder, A., 2009, Physics, Formation and Evolution of Rotating Stars (Springer)

Moravveji, E., Aerts, C., Pápics, P.I., Triana, S.A., \& Vandoren, B., 2015, A\&A, 580, A27

Niemela, J.J., Skrbek, L., Sreenivasan, K.R., \& Donnelly, R.J., 2000, Nature, 404, 837

Rieutord, M., 2015, Fluid Dynamics: An Introduction (Springer), p. 508 
Rieutord, M. \& Zahn, J.-P., 1995, A\&A, 296, 127

Roxburgh, I.W., 1989, A\&A, 211, 361

Saslaw, W.C., \& Schwarzschild, M., 1965, ApJ, 142, 1468

Spiegel, E.A., 1971, Ann. Rev. Astron. Astrophys., 9, 323

Tsuji, Y., Mizuno, T., Mashiko, T., \& Sano, M., 2005, Phys. Rev. Lett., 94, 034501 Vassilicos, C., 2015, Ann. Rev. Fluid Mech., 47, 95

Zahn, J.-P., 1991, A\&A, 252, 179 